PRAMANA
$\begin{gathered}\text { journal of } \\ \text { physics }\end{gathered}$$\quad \begin{aligned} & \text { Vol. } x x, \text { No. } x \\ & \text { xxxxxxx } x x x x \\ & \text { pp. } 1-9\end{aligned}$

\title{
Instantaneous frequencies of a chaotic system
}

\author{
C. Chandre ${ }^{1}$ and T. Uzer ${ }^{2}$ \\ ${ }^{1}$ Centre de Physique Théorique*, CNRS Luminy, Case 907, F-13288 Marseille Cedex 9, \\ France \\ ${ }^{2}$ Center for Nonlinear Science, School of Physics, Georgia Institute of Technology, Atlanta, \\ Georgia 30332-0430
}

\begin{abstract}
The structure and geometry of high-dimensional, complex dynamical systems is usually hidden under a profusion of numerical data. We show that time-frequency analysis allows one to analyze these data regardless of the number of degrees of freedom. Our method takes snapshots of the system in terms of its instantaneous frequencies defined as ridges of the time-frequency landscape. Using the wavelet transform of a single trajectory, it can characterize key dynamical properties like the extent of chaos, resonance transitions and trappings.
\end{abstract}

Keywords. Time-frequency analysis,wavelets, Hamiltonian systems

PACS Nos 05.45.-a,32.80.Rm

\section{Introduction}

Multi-dimensional, complex systems tend to overwhelm the researcher with data: think of fluid dynamical data from the oceans or the millions of trajectories that today's powerful computers can generate in an instant. It is not only computers that generate reams of data of complex dynamical systems, but in many areas of science and engineering, experimental techniques to observe real-time dynamical phenomena have also developed at a pace far more rapid than the theory required to make sense of such data. In this article we show how to break through this thicket, regardless of the number of degrees of freedom, by time-frequency analysis [1].

For low dimensional systems, the geometric framework of dynamical systems theory gives a way of understanding these data. This point of view, due to Poincaré, asks about the relationship between all possible trajectories, rather than the evolution of individual trajectories. This leads immediately to the notion of phase space

*Unité Mixte de Recherche (UMR 6207) du CNRS, et des universités Aix-Marseille I, Aix-Marseille II et du Sud Toulon-Var. Laboratoire affilié à la FRUMAM (FR 2291). 
structure as a key notion for making sense of the many and varied regimes that a nonlinear dynamical system can exhibit.

In this paper, we present a tool to penetrate the dynamics of such systems through the geometrical framework of dynamical systems theory, and without resorting to dimensional reduction schemes with their attendant flaws and loss of information. Our approach [1] bridges the gap between the high-dimensional complex systems world and the tried-and-true techniques which have provided so much insight into low-dimensional, near-integrable systems. The latest developments of time-frequency analysis of signals, of which the wavelet transform is but one example [2], form the cornerstone of our method. The advantages of the time-frequency representation are that it does not require near integrability or action-angle like variables, and it works for dynamical systems regardless of their number of degrees of freedom.

Technically speaking, our method extracts the frequencies of a chaotic system based on wavelet decomposition. Frequencies and especially resonances between them form the basis of our understanding of nonlinear systems (perturbation theorems, intramolecular dynamics [3,4], Laskar's work in celestial mechanics [5-7] and particle accelerators [8]). For quasi-periodic and weakly chaotic systems, the frequency representation has been revealing because it shows how motion can be trapped in some resonance zones associated with quasi-periodicity. These successes suggest that snapshots of a chaotic system in terms of frequencies should be much more revealing than other representations, a worthwhile goal which, until now, has not been achieved. Indeed, the interpretation of a frequency in rapidly-changing systems is an old problem full of subtleties $[9,10]$. The information resulting from our work is much richer than the methods devised to distinguish between regular and chaotic orbits : Most of the standard dynamical systems diagnostics, e.g., Lyapunov exponents, entropy, fractal dimensions, etc., have rigorous mathematical definitions in the infinite-time limit. Asymptotic quantities are not practical for many important problems since they do not reflect the history and local properties of the trajectory. Instead, our method gives a snapshot of the dynamics at a fixed instant of time, detecting resonance trappings and transitions, and characterizes weak and strong chaos in many-dimensional systems [11]. We will show below how this information can be directly correlated with phase space structure.

We associate a set of time-varying frequencies with the trajectory by decomposing it on a set of elementary functions, the wavelets, which are localized in time and frequency. Wavelet decomposition has been used to analyze trajectories of Hamiltonian systems in celestial mechanics [12] and in molecular dynamics $[13,14]$. In Refs. [12,14], the main frequency was extracted by computing the frequency curve where the modulus of the wavelet transform is maximum. However, stopping at this maximum frequency can give misleading or wrong information about resonance transitions (see Ref. [1] for more details). Instead, we have found that the relevant information of the trajectory is contained in the so-called ridges of the landscape formed by the magnitude of the coefficients of the time-frequency decomposition [15]. A ridge curve (which has a certain time length) corresponds to time-frequency energy localization and is also called instantaneous frequency curve.

The extraction of instantaneous frequencies is illustrated by using the trajectories of the standard map. This well-known map provides a benchmark for the method 
since its phase space structures are well known. We will show how our method constitutes a powerful tool to reveal phase space structures using instantaneous frequencies coming from single trajectory analysis, and is therefore, ideally suited for the analysis of high-dimensional systems.

Given a real signal $f(t)$ which can be, e.g., one coordinate of the system, two methods are used to compute a time-frequency (denoted $u-\xi$ ) representation of $f$ : window Fourier transform and continuous wavelet transform.

\section{Windowed Fourier transform}

The windowed Fourier transform (also called Gabor transform) of $f(t)$ is given by

$$
S f(u, \xi)=\int_{-\infty}^{+\infty} f(t) g(t-u) e^{-i \xi t} d t
$$

where the window $g$ is chosen to be a Gaussian window

$$
g(t)=e^{-t^{2} / 2 \sigma^{2}} /\left(\sigma^{2} \pi\right)^{1 / 4} .
$$

In Laskar's work $[5,6]$, the Hanning filter is chosen rather than a Gaussian filter. The Hanning filter has the advantage of having a finite support whereas the Gaussian filter has an optimal time-frequency resolution. In what follows, we will consider an energy density called a spectrogram

$$
P_{S} f(u, \xi)=|S f(u, \xi)|^{2} .
$$

\section{Continuous wavelet transform}

The continuous wavelet transform of $f(t)$ gives a time-scale representation of the trajectory and is given by

$$
W f(u, s)=\frac{1}{\sqrt{s}} \int_{-\infty}^{+\infty} f(t) \psi^{*}\left(\frac{t-u}{s}\right) d t
$$

where the mother wavelet $\psi$ is chosen to be a Gabor (modulated Gaussian) wavelet, also called Morlet-Grossman wavelet:

$$
\psi(t)=e^{i \eta t} e^{-t^{2} / 2 \sigma^{2}} /\left(\sigma^{2} \pi\right)^{1 / 4} .
$$

The transform depends on $\eta$ which is the center frequency of the wavelet. The time-frequency representation is obtained by the relation between the scale $s$ and the frequency $\xi: \xi=\eta / s$. We will consider the normalized scalogram

$$
\frac{\xi}{\eta} P_{W} f(u, \xi)=\frac{1}{s}|W f(u, s)|^{2}
$$

which can be interpreted as the energy density in the time-frequency plane.

Pramana - J. Phys., Vol. xx, No. x, $\mathrm{xxxxxxxx} \mathrm{xxxx}$ 
For a periodic trajectory $f(t)=e^{i \omega t}$, the spectrogram

$$
P_{S} f(u, \xi)=2 \sigma \sqrt{\pi} e^{-\sigma^{2}(\omega-\xi)^{2}}
$$

and the normalized scalogram $\xi \eta^{-1} P_{W} f(u, \xi)=2 \sigma \sqrt{\pi} e^{-\sigma^{2} \eta^{2}(\omega / \xi-1)^{2}}$ are maximum for $\xi=\omega$, independently of the time $u$. For a quasi-periodic trajectory $f(t)=\sum_{k} A_{k} e^{i \omega_{k} t}$, the spectrogram and the normalized scalogram present a sum of localized peaks and interference terms : The wavelet transform of $f$ is given by the sum of the wavelet transform of $A_{k} e^{i \omega_{k} t}$ since the wavelet transform is linear. Therefore, the normalized scalogram is a sum of peaks located at $\xi=\omega_{k}$ and of width proportional to $\omega_{k} /(\eta \sigma)$, with some additional interference terms of the form

$$
A_{l} A_{k}^{*} e^{-\sigma^{2} \eta^{2}\left(\omega_{l} / \xi-1\right)^{2} / 2} e^{-\sigma^{2} \eta^{2}\left(\omega_{k} / \xi-1\right)^{2} / 2} e^{i\left(\omega_{l}-\omega_{k}\right) u} .
$$

The effect of these terms is visible if $(a)$ at least two amplitudes $A_{k}$ and $A_{l}$ are large enough, and $(b)$ if the difference of their frequencies $\omega_{l}-\omega_{k}$ is smaller than $\omega_{l} /(\eta \sigma)$. In order to distinguish two frequency components $\omega_{l}$ and $\omega_{k}$ of amplitude $A_{k}$ and $A_{l}$ of the same order, the parameters of the wavelet have to be chosen according to the condition

$$
\eta \sigma \geq\left(\omega_{l}+\omega_{k}\right) /\left|\omega_{l}-\omega_{k}\right|
$$

The various main frequencies of the trajectory can be obtained by looking at the ridges of the spectrogram or normalized scalogram, also called instantaneous frequencies. These ridges are local maxima with respect to the frequency $\xi$, of the energy density in the time-frequency plane. For instance, a periodic trajectory has only one constant ridge $\xi=\omega$ and a quasi-periodic trajectory has approximately a set of constant ridges (if the interferences are negligible) at $\xi=\omega_{k}$. Ridges of sufficiently high amplitude are detected by the method.

Both these methods have time-frequency resolution limitations for the determination of the instantaneous frequencies. The windowed Fourier transform relies on an a priori choice of length of the window $\sigma$. Any event (trapping, transition, etc.) happening on short time scales (less than $\sigma$ ) or with small frequencies (less than $\sigma^{-1}$ ) is missed by this method. On the contrary, the main advantage of wavelets is that it follows the rapid variations of the instantaneous frequencies since it adapts the length of the window according to the frequency [2]. This is due to the fact that the compact support of the wavelet is proportional to $\eta \sigma / \omega$. Therefore the time span is larger for small frequencies than for large ones. For this reason we choose the wavelet basis for the extraction of there instantaneous frequencies.

\section{Application to the standard map}

In order to illustrate the notion of instantaneous frequencies, we consider a wellknown two-dimensional symplectic map, namely the standard map $(x, y) \mapsto\left(x^{\prime}, y^{\prime}\right)$ : 


$$
\begin{aligned}
& x^{\prime}=x-a \sin y \\
& y^{\prime}=x^{\prime}+y \bmod 2 \pi .
\end{aligned}
$$

The phase space structures of this two-dimensional map are well-known. We use this simple model to show that these structures are revealed by the wavelet analysis of a single trajectory, i.e., by the computation of the associated instantaneous frequencies.

In what follows, we use the routines (fast algorithms) of WaveLab [16]. In the numerical implementation, we use $\eta=5$ and $\sigma=4$. We analyze a single real signal which is the $y$-coordinate. Analogous results can be obtained by analyzing the $x$-coordinate or another function of these two coordinates. This point is one of the main motivations for the analysis of many dimensional systems, provided they are highly nonlinear and strongly coupled (which are precisely the cases where there is no method for analysis): The analysis of the phase space structures can be obtained from a single signal.

Quasi-periodic trajectory - We consider a trajectory of the standard map for $K=0.7$ starting at $x_{0}=4.1$ and $y_{0}=0$ over a time span of $T=12868$. This trajectory, plotted in the first panel of Fig. 1, corresponds to a smooth invariant torus. The main frequency is (approximately) equal to $\omega_{0}=(3-\sqrt{5}) / 2$. Figure 1 shows the different ridges of the normalized scalogram. These ridges are approximately constant in time, as one would expect. The upper curve represents the main frequency $\omega_{0}$, i.e. the ridge curve that has the strongest coefficient in the normalized scalogram. The other two main frequency curves (whose amplitude is at least one tenth of the maximum) are located at $\omega_{1}=1-2 \omega_{0}$ and $\omega_{2}=3 \omega_{0}-1$.

We can also see the interferences (oscillations around the expected frequency) between these frequencies. In order to reduce these oscillations, we can increase the parameter $\eta \sigma$.

A similar picture is obtained for the ridges of the spectrogram. The accuracy in determining these frequencies is limited (of order $\Delta \omega / \omega \sim 10^{-2}-10^{-3}$ mainly because we use the fast-algorithm wavelet transform), and Laskar's frequency analysis [17] (which computes the maximum frequency of the filtered Fourier transform on the whole time interval $[0, T]$ and a Gram-Schmidt orthogonalization procedure to compute secondary frequencies) is more suited for this purpose and much more accurate (with a Hanning filter, proportional to $T^{-4}[6]$ ).

Weakly chaotic motion - We consider a trajectory obtained for the standard map with $K=1.1$ and initial conditions $x_{0}=4.25$ and $y_{0}=0$. We analyze this weakly chaotic trajectory over the time interval of total length $T=12868$. Figure 2 depicts the main ridge (the secondary ridges are less than one half of the maximum in amplitude) and shows clearly different trappings as time evolves: for instance, the long trapping which occurs in the 1:3 resonance for for $4500 \leq t \leq 8000$, or in $1: 4$ for $9300 \leq t \leq 9800$. These trappings result from the trajectory passing nearby islands surrounding elliptic periodic orbits (the other ridges contain the secondary frequencies of the nearby quasi-periodic motion). The transitions between these resonances occur quite smoothly, passing by other type of resonance trappings. The transitions between different resonances occur when the chaotic trajectory passes nearby a hyperbolic point where great variations of the frequencies are expected. The trajectory is weakly chaotic since the main information can be obtained from a single connected instantaneous frequency curve. In this regime, looking at the 
frequency where the spectrogram or scalogram is maximum is meaningful. Figure 3 represents the same trajectory as in Fig. 2 on a smaller time-span and with a smaller ridge detection threshold. It represents a transition between a trapping near a borken invariant rotational torus (cantorus) characterized by the same frequencies as in Fig. 1 to a resonance trapping in the 1:3 resonance. This transition occurs by the merger of the two main frequencies of the quasiperiodic motion. The Poincaré section of this segment of trajectory represented on the upper panel is consistent with this transition.

Resonance transitions - Apart from this type of transition, the time-frequency resolution of wavelets allows us to analyze the mechanism of resonance transition. Figure 4 shows a ridge plot obtained for $K=2, x_{0}=1$ and $y_{0}=0$ on a time length of $T=3000$. The maximum frequency (characterized by the frequency where the wavelet coefficients are maximum) jumps discontinuously from one ridge to another. However, by looking at secondary ridges, the mechanism appears different: A second ridge increases in amplitude while the first ridge decreases in amplitude. On some time interval, two main ridges are constant in time and of the same order in amplitude. Then the first ridge disappears and the second ridge becomes the dominant one. This situation is typical from other ridge plots of trajectories showing transitions from ridges and hence resonance transitions. Thus we have a new characterization of resonance transition that applies in more general situations. Resonance transitions are a manifestation of the fulfillment of the Chirikov resonance overlap criterion [18]. In this sense, our method extends this well-known criterion for transition to chaos to high-dimensional dynamical systems that are far from integrable.

When $K$ increases, the trajectories are more and more chaotic (filling a bigger part of phase space). More and more important instantaneous frequency curves appear, and in those cases, looking at the maximum frequency leads to erroneous results as shown above since several ridges have the same amplitude and the information cannot be reduced to (or deduced from) a single instantaneous frequency.

Strongly chaotic motion - We consider a strongly chaotic trajectory of the standard map obtained with $K=5$ and initial conditions $x_{0}=1$ and $y_{0}=0$. Figure 5 shows the ridge plot obtained from the normalized scalogram. It shows that no trappings occur in this time interval and that the transitions between ridges occur as explained above. The presence of a lot (but a finite number) of ridges reflects the broad-band spectrum of a chaotic trajectory. These pictures lead to a characterization of weak and strong chaos by looking at the number of important ridges in the system: Weak chaos is characterized by one main connected instantaneous frequency curve whereas strong chaos is characterized by multiple short ridges (the number of them increases as $K$ increases).

\section{Conclusions}

In summary, we showed how instantaneous frequencies reveal the phase space structures (resonance transitions, trappings, etc.) of a well-known chaotic system. Our method based on a single trajectory analysis is therefore very well suited for exploring phase space structures of systems with high number of degrees of freedom. 
It uses the geometrical framework of dynamical systems theory for the purpose of understanding the various possibilities for the evolution of trajectories in highdimensional, complex dynamical systems. Consequently, it should become a useful tool not only for the analysis of trajectories generated by computers, but also for trajectories generated in experiments.

\section{References}

[1] C. Chandre, S. Wiggins, and T. Uzer, Physica D 181, 171 (2003).

[2] S. Mallat, A wavelet tour of signal processing (Academic Press, San Diego, 1999).

[3] C.C. Martens, M.J. Davis, and G.S. Ezra, Chem. Phys. Lett. 142, 519 (1987).

[4] J. von Milczewski and T. Uzer. Mapping multidimensional intramolecular dynamics using frequency analysis. In D.L. Thompson, editor, Modern Methods for Multidimensional Dynamics Computations in Chemistry, pages 190 - 200, Singapore, 1998. World Scientific.

[5] J. Laskar, Icarus 88, 266 (1990).

[6] J. Laskar, in Hamiltonian Systems with Three or More Degrees of Freedom, NATO ASI Series, edited by C. Simó (Kluwer Academic Publishers, Dordrecht, 1999), pp. $134-150$.

[7] A. Correia and J. Laskar, Nature 411, 767 (2001).

[8] D. Robin, C. Steier, J. Laskar, and L. Nadolski, Phys. Rev. Lett. 85, 558 (2000).

[9] L. Mandel, Am. J. Phys. 42, 840 (1974).

[10] P. J. Loughlin, IEEE Signal Processing Letts. 4, 123 (1997).

[11] E. Shchekinova, C.Chandre, Y. Lan, and T. Uzer, J. Chem. Phys. 121, 3471 (2004).

[12] T.A. Michtchenko and D. Nesvorny, Astron. Astrophys. 313, 674 (1996).

[13] A. Askar, A.E. Cetin, and H. Rabitz, J. Phys. Chem. 100, 19165 (1996).

[14] L.V. Vela-Arevalo and S. Wiggins, Int. J. Bifurcat. Chaos 11, 1359 (2001).

[15] N. Delprat et al., IEEE Trans. Inform. Theory 38, 644 (1992).

[16] http://www-stat. stanford.edu/ wavelab.

[17] J. Laskar, C. Froeschlé, and A. Celletti, Physica D 56, 253 (1992).

[18] B.V. Chirikov, Phys. Rep. 52, 263 (1979). 

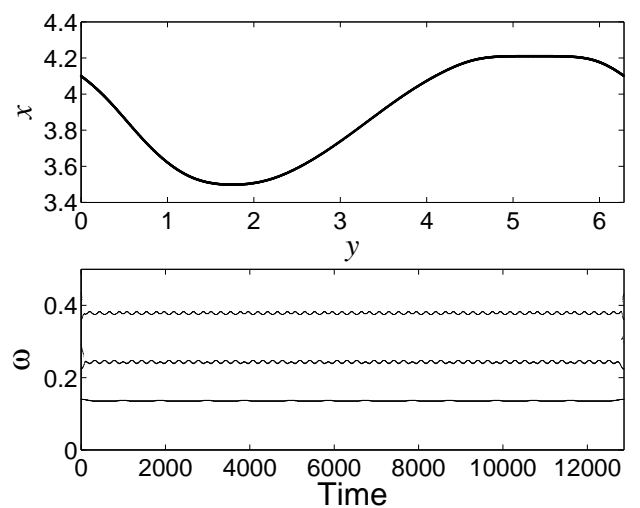

Figure 1. Phase portrait (first panel) and ridge plot (second panel) of a quasi-periodic trajectory of the standard map obtained for $K=0.7$ and initial conditions $x_{0}=4.1$ and $y_{0}=0$.
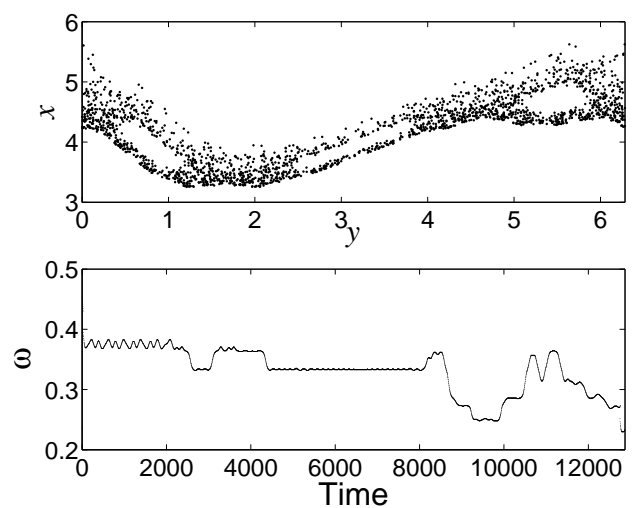

Figure 2. Phase portrait (first panel) and ridge plot (second panel) of a weakly chaotic trajectory of the standard map obtained for $K=1.1$ and initial conditions $x_{0}=4.25$ and $y_{0}=0$.
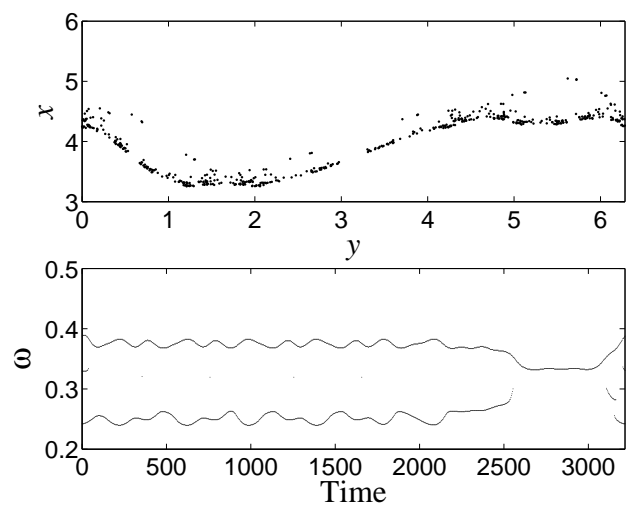

Figure 3. Phase portrait (first panel) and ridge plot (second panel) of a weakly chaotic trajectory of the standard map obtained for $K=1.1$ and initial conditions $x_{0}=4.25$ and $y_{0}=0$.

Pramana - J. Phys., Vol. xx, No. x, $\mathrm{xxxxxxxx} \mathrm{xxxx}$ 

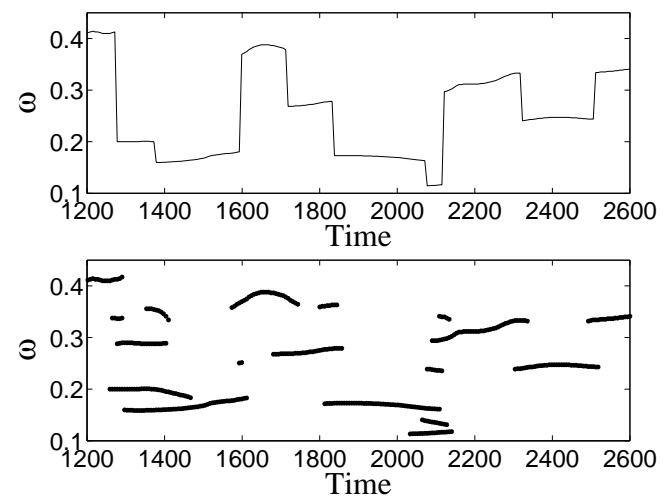

Figure 4. Resonance transition: Ridge plot of a trajectory of the standard map with $K=2$ and initial conditions $x_{0}=1$ and $y_{0}=0$.

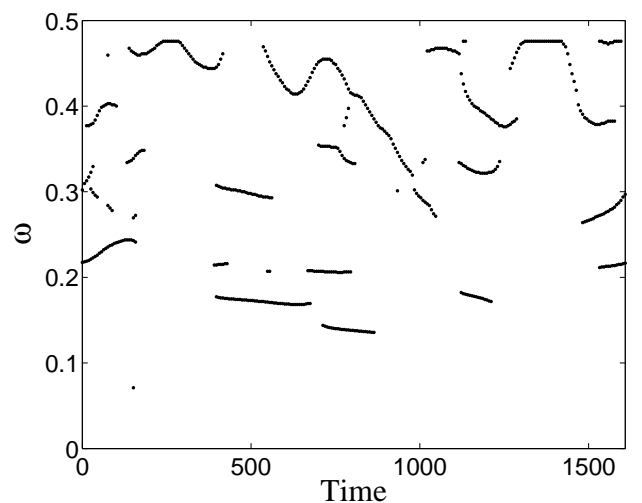

Figure 5. Ridge plot of a strongly chaotic trajectory of the standard map with $K=5$ and initial conditions $x_{0}=1$ and $y_{0}=0$. 